ARTICLE

\title{
Light-activated shape morphing and light-tracking materials using biopolymer-based programmable photonic nanostructures
}

\author{
Yu Wang (1) 1,2,9, Meng Li (10 1,2,9, Jan-Kai Chang3 ${ }^{3}$, Daniele Aurelio4, Wenyi Li1,2, Beom Joon Kim (1) ${ }^{1,2}$, \\ Jae Hwan Kim³, Marco Liscidini ${ }^{4}$, John A. Rogers (1) ${ }^{3,5}$ \& Fiorenzo G. Omenetto (1) 1,2,6,7,8凶
}

Natural systems display sophisticated control of light-matter interactions at multiple length scales for light harvesting, manipulation, and management, through elaborate photonic architectures and responsive material formats. Here, we combine programmable photonic function with elastomeric material composites to generate optomechanical actuators that display controllable and tunable actuation as well as complex deformation in response to simple light illumination. The ability to topographically control photonic bandgaps allows programmable actuation of the elastomeric substrate in response to illumination. Complex three-dimensional configurations, programmable motion patterns, and phototropic movement where the material moves in response to the motion of a light source are presented. A "photonic sunflower" demonstrator device consisting of a light-tracking solar cell is also illustrated to demonstrate the utility of the material composite. The strategy presented here provides new opportunities for the future development of intelligent optomechanical systems that move with light on demand.

\footnotetext{
${ }^{1}$ Silklab, Suite 4875, 200 Boston Avenue, Tufts University, Medford, MA, USA. ${ }^{2}$ Department of Biomedical Engineering, Tufts University, Medford, MA, USA.

${ }^{3}$ Center for Bio-Integrated Electronics, Northwestern University, Evanston, IL, USA. ${ }^{4}$ Dipartimento di Fisica, Università degli Studi di Pavia, Pavia, Italy.

${ }^{5}$ Departments of Materials Science and Engineering, Biomedical Engineering, Neurological Surgery, Chemistry, Mechanical Engineering, Electrical Engineering and Computer Science, and Simpson Querrey Institute for BioNanotechnology, Northwestern University, Evanston, IL, USA. ${ }^{6}$ Department of Physics, Tufts University, Medford, MA, USA. ${ }^{7}$ Department of Electrical Engineering, Tufts University, Medford, MA, USA. ${ }^{8}$ Laboratory for Living Devices, Tufts University, Medford, MA, USA. ${ }^{9}$ These authors contributed equally: Yu Wang, Meng Li. ${ }^{凶}$ email: Fiorenzo.Omenetto@tufts.edu
} 
ight-matter interactions are sophisticatedly controlled by natural systems for efficient light harvesting, manipulation, and management utilizing micro-and nano-structural constructs built from a limited choice of constituent elements ${ }^{1,2}$. Functional outcomes include structural coloration/camouflage, vision, signaling, communication, thermal regulation, and photosynthesis, through effective manipulation of light reflection, diffusion, diffraction, transmission, and absorption ${ }^{3-5}$. These architectures have long been a source of inspiration for the development of multiple artificial optical functional materials for effective light-energy conversion ${ }^{2,3,6}$. Specifically interesting among these are periodic nanostructures (i.e., photonic crystals - PhCs) that can control and manipulate the flow of light by strongly suppressing the propagation of photons of specific frequencies through the structure ${ }^{7,8}$. These geometries have been successfully utilized as light-harvesting layers in various optical devices to enhance lightenergy conversion, including photocatalysis ${ }^{9,10}$, photovoltaics ${ }^{9,11}$, light-emitting diodes ${ }^{12,13}$, and photothermal systems ${ }^{14,15}$.

Another light-matter interaction of interest converts optical energy into mechanical action, with several outcomes that add utility to applications in soft robotics ${ }^{16,17}$, biomedical devices ${ }^{18,19}$, or sensing ${ }^{20}$ because of the ability to remotely activate devices ${ }^{21}$. There has been exciting progress in the development of high performance optomechanical systems based on liquid crystal networks ${ }^{22-24}$, hydrogels ${ }^{25,26}$, shape memory polymers ${ }^{27,28}$, inequivalent expansion of gradient materials ${ }^{29,30}$, inorganic materials $^{31,32}$, and others ${ }^{33}$. Current strategies for materials for optomechanical control involve the optimization of molecular structure assembly with outcomes that cover complex movement such as folding ${ }^{23,30}$, swimming $25,34,35$, walking ${ }^{31}$, self-oscillation $^{24,25}$, and heliotropic motion ${ }^{26,36}$. Great efforts have been devoted in finding various approaches based on photothermal and photochemical mechanisms to manage the light-energy conversion that dominate the actuation $22,29,34,37$. Most optomechanical devices are engineered to perform local light-energy conversion $23,30,31$, which usually involve complex and energyintensive fabrication process or complicated setups.

We describe here a composite material exploiting both modes of light-matter interaction in which the confluence of programmable photonic crystals with elastomeric materials enables a class of photo-responsive actuators that allow controllable and tunable actuation, and complex deformation in response to simple light illumination.

\section{Results}

Photonic optomechanical actuator. The photonic actuator is a bimorph structure consisting of a silk inverse opal (SIO), doped with gold nanoparticles and polydimethylsiloxane (PDMS), as illustrated in Fig. 1a. Silk fibroin is chosen as the passive layer because of its versatility, flexibility, ease of functionalization, remarkable optical properties, nanoscale processability, and polymorphic features that allow for photonic crystal programmability ${ }^{38,39}$. Silk also possesses a negative coefficient of thermal expansion (CTE) ${ }^{40}$ which is suitable to interface with PDMS as the active layer because of its large coefficient of thermal
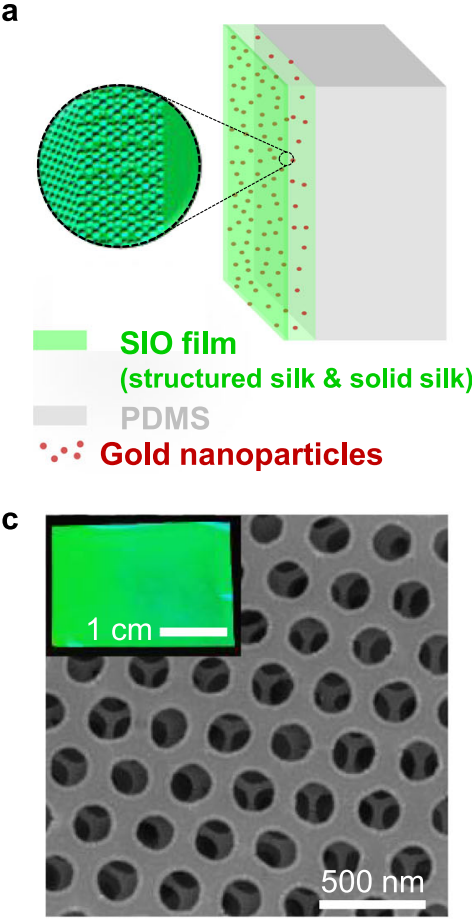

b

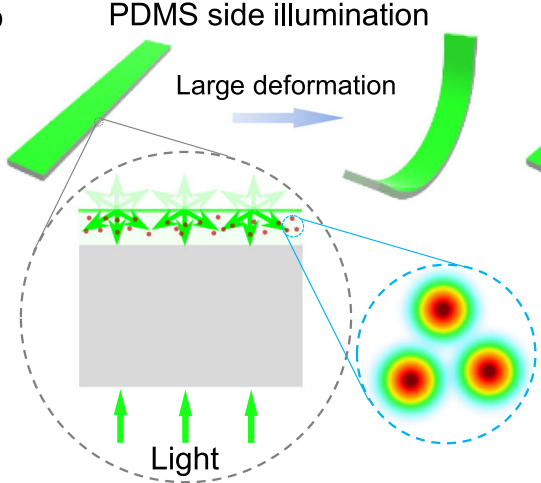

d
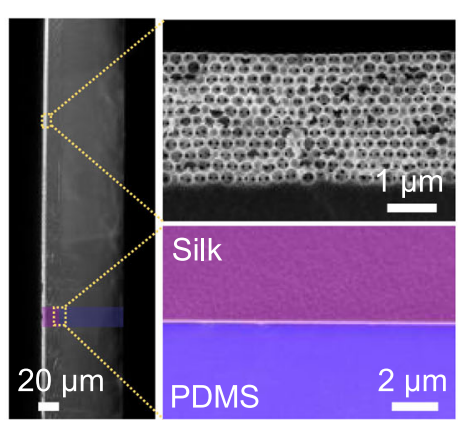

e
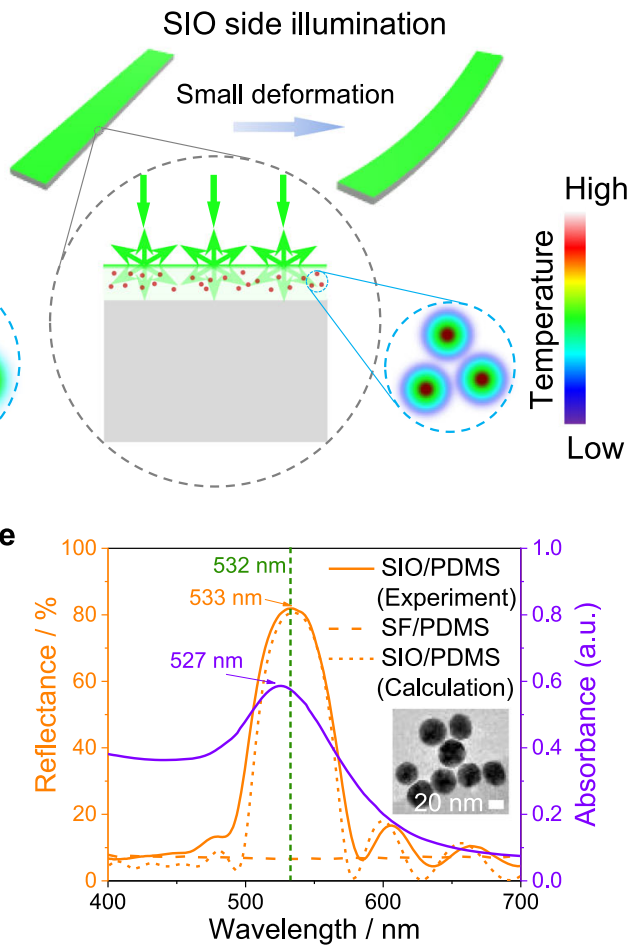

Fig. 1 Structure, mechanism, and optical properties of photonic optomechanical actuator. a Schematic illustration of the structure of photo-deformable photonic bilayer film. The enlarged image shows the nanostructure of the inverse opal. b Schematics showing the mechanism of photonic-structurecontrolled actuation. The photonic structure enhances (PDMS side illumination) or weakens (SIO side illumination) the gold-nanoparticles-driven photothermal conversion by controlling light propagation within the system. c Surface SEM image of SIO obtained from the colloidal crystals composed of polystyrene spheres with a diameter of $300 \mathrm{~nm}$. The inset is a photograph of the as-prepared photonic bilayer film, showing bright green iridescence. The image was collected in the direction perpendicular to the SIO film. d Cross-sectional SEM images of the photonic bilayer film. The enlarged images clearly show the photonic crystal structure displayed by ordered, hollow silk fibroin structure with air holes on the wall (top) and the compactly contacted interface between silk and PDMS (bottom). e Measured and simulated reflectance spectra of the photonic bilayer film and absorbance spectrum of gold nanoparticles. The experimental curve is in agreement with the theoretical calculation. Inset shows a transmission electron microscopy image of the gold nanoparticles. Data for an unstructured SF/PDMS bilayer film is also shown for comparison. 
expansion, and excellent durability against repeated deformation and high temperature ${ }^{41}$, on top of its optical transparency. The significant CTE difference between silk fibroin ${ }^{40}$ and PDMS $^{41}$ allows for the generation of bending motion in response to temperature increases caused by the photothermal effect when the bimorph is exposed to light.

The formation of programmable SIO films is based on colloidal assembly of polystyrene nanosphere multilayers as templates as previously described $38,42,43$. Silk solution doped with gold nanoparticles is infiltrated in the template and allowed to solidify into a free-standing silk/polystyrene composite film. The resulting structure obtained after dissolving the polystyrene spheres in toluene consists of a free-standing gold-nanoparticle-doped SIO film with a nanostructured silk layer (enlarged image in Fig. 1a). The gold nanoparticles are mostly located in the solid part of the SIO film because of the dimensional constraints imposed by the nanostructure that are comparable to the dimensions of gold nanoparticles $(>30 \mathrm{~nm}$ in diameter), which is crucial for the generation of direction-dependent deformation as described below. The bimorph structure is then prepared by casting the PDMS onto the flat side of the film via spin coating and subsequent drying.

The nanostructured silk layer provides a photonic crystal lattice layer which serves as an effective reflector, enhancing or weakening the interaction between gold nanoparticles and light as a function of its direction of incidence (Fig. 1b). This photonic layer only works when its stop-band matches the plasmonic absorption of gold nanoparticles. When illuminating the SIO/ PDMS bilayer film from PDMS side, light with wavelength matching the stop-band is partially reflected back into the goldnanoparticle-rich layer after reaching the thin nanostructured layer, leading to a longer light propagation path within the system and, thus, stronger interaction between gold nanoparticles and light. This causes larger deformation (Fig. 1b, left) when compared to films with totally mismatched stop-band or without photonic layer. By contrast, light incident from SIO side is partially reflected and does not penetrate the gold-nanoparticle-rich layer, resulting in weaker interaction between gold nanoparticles and light and, thus, less material deformation (Fig. 1b, right).

In order to maximize light-matter interaction the photonic crystal layer is designed to have a stop-band that is spectrally matched to the absorption peak of gold nanoparticles. Reflectivity of the photonic structure is increased by controlling its lattice constant and the number of nanosphere layers. The resulting structure is shown in Fig. 1c, d. A scanning electron microscopy (SEM) image of the surface of the SIO shows the highly ordered hexagonal arrays of air cavities with lattice constant (center-tocenter distance of the air cavities, $\Lambda$ ) of $300 \mathrm{~nm}$ (Fig. 1c), while the cross-sectional SEM image of SIO shows twelve-layer highly ordered hollow silk fibroin spheres with air holes on the wall (Fig. 1d) and confers the SIO a bright green iridescent color. Analysis of the interface between silk and PDMS reveals uniform contact, thereby guaranteeing effective heat transfer and stable performance. The reflectance spectrum of SIO taken at normal incidence shows a narrow stop-band peak centered at $\lambda=533 \mathrm{~nm}$ (Fig. 1e), which is well matched to the absorbance peak of doped gold nanoparticles $(\lambda=527 \mathrm{~nm})$. The reflectance intensity of the stop-band peak is shown to approach $80 \%$, thus enabling higher discrimination between light propagating within the device and interacting with gold nanoparticles than when traversing a portion without an inverse opal structure and PDMS (SF/PDMS). This is confirmed by analyzing the photothermal conversion of the bilayers (Supplementary Fig. 1). As expected, illuminating the photonic bilayer film on the PDMS side and SIO side shows high and low temperature increase (and corresponding photothermal conversion efficiency), respectively, while the SF/PDMS sample shows intermediate values and no dependence on the illumination direction. Unless otherwise specified, all the SIOs considered here are twelve-layered inverse opals with $\Lambda=300 \mathrm{~nm}$ (i.e., green colored SIOs).

Actuation performance of photonic bilayer. To evaluate lightinduced deformations mediated by the photonic crystals on the bilayer films, small strips measuring $25 \times 2 \mathrm{~mm}$ were fixed at one end and exposed to light. A green laser $(\lambda=53 \mathrm{~nm})$ was used as the light source and directed perpendicularly to the sample surface (i.e., $\theta=0^{\circ}$, Supplementary Fig. 2), unless otherwise noted. The strip illuminated on the PDMS side bends significantly more than when illuminated on the SIO side (Fig. 2a). Figure $2 b$ plots the measured displacement at different illumination intensities. The displacement is defined as the straight distance at which the strip tip travels (Supplementary Fig. 3). Consistent with the results of heating (Supplementary Fig. 1a), PDMS side illumination on SIO/PDMS exhibits the largest displacement, while SIO side illumination shows the least. In comparison, SF/PDMS samples show intermediate deformation and the illumination direction does not affect the displacement. The evaluation of displacement induced by the laser illumination time was also examined (Fig. 2c). The displacement follows an exponential function when the laser is switched on and off. The response rate is evaluated from the initial slope of the displacement curve as a function of time after the light is turned on (Fig. 2d). SIO/PDMS samples with PDMS side illumination show the fastest response rate, while SIO side illumination gives the slowest response rate. The actuation of the photonic bilayer film is reversible with no observable deterioration in displacement after 100 cycles (Fig. 2e).

Programmable shape-morphing. While the ability to modulate actuation with the photonic structure is remarkable, the capability to design and control the geometric distribution of its photonic bandgap (position and intensity) provides further degrees of control over optomechanical actuation of the photonic bilayer film. This is first demonstrated by constructing SIOs with different nanostructures by either adjusting the number of layers and lattice constant of assembled colloidal crystals or by reconfiguring the photonic lattices after the formation of the inverse opal structure ${ }^{38}$ (Supplementary Fig. 4). For instance, Watervapor-treatment induces the irreversible compression of photonic lattice in the weak vertical direction of green-colored SIO, leading to the modulation of the photonic stop-band and, thus, the decrease (PDMS side irradiation) or increase (SIO side irradiation) in the displacement (Fig. 3a). Such approach leverages the ability of water vapor to direct the polymorphic transitions of amorphous silk fibroin and provides the ability to design patterned photonic lattices with different responses ${ }^{38,39}$. Evaluation of the bilayer's optical response shows that the displacement is linearly correlated with the reflected intensity at $532 \mathrm{~nm}$ (i.e., the wavelength of the laser source), independently of the SIO structure (Fig. 3b). The displacement of PDMS side illumination increases with the increase of reflectance, while it is just the reverse for SIO side illumination.

This photonic bandgap-dependent actuation enables the construction of complex 3D configurations by optically inducing controlled deformation of different 2D structures. As a first demonstration, a flower-like geometry was assembled using bilayers with different photonic lattices: a stamen made of a yellow SIO and six petals made of green and blue-violet SIOs (Fig. 3c). When the construct is illuminated from SIO side, the blue-violet petals bend dramatically towards the light source 
a
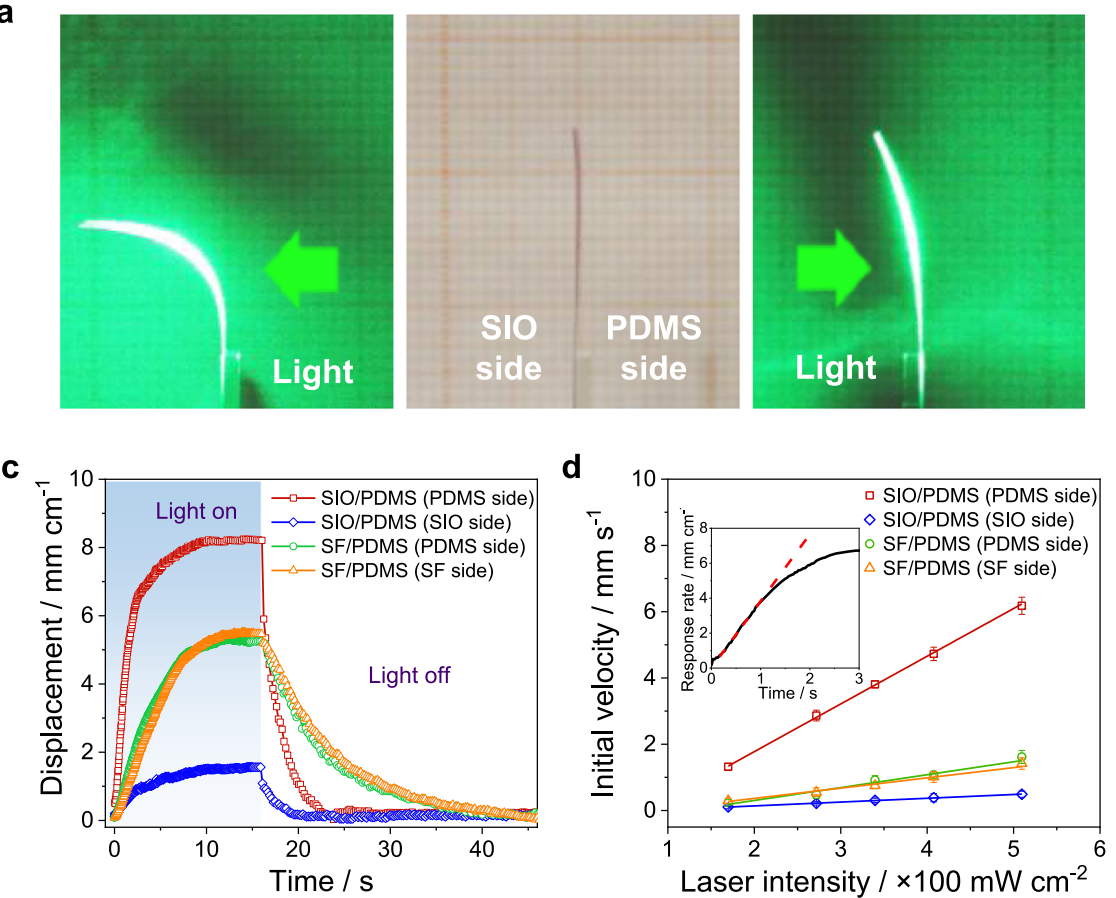
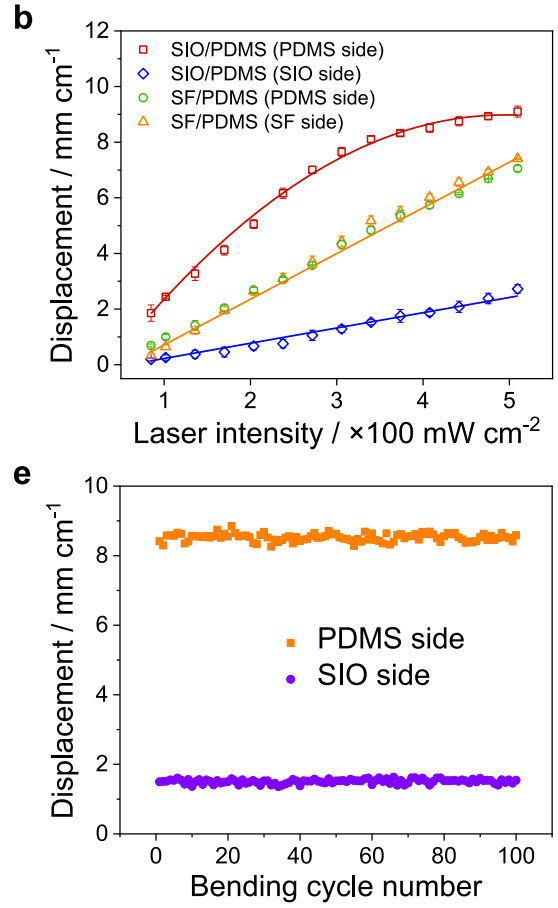

Fig. 2 Actuation performance of the photonic bilayers. a Deformation of a photonic bilayer strip $(20 \times 2 \mathrm{~mm})$ in response to a green laser $(I=17 \mathrm{~m} \mathrm{~cm}-2)$. b Dependence of maximal displacement on laser intensity. $\mathbf{c}$ Dependence of displacement on the laser illumination time $(I=35 \mathrm{~m} \mathbf{c m}-2)$. $\mathbf{d}$ Response rate of bilayers in the initial deformation stage as a function of laser intensity. Inset shows the calculation of response rate, which is defined as the slope of the tangent line. e Dependence of the displacement on the bending cycle number of photonic bilayer film $\left(I=35 \mathrm{~m} \mathrm{~cm}^{-2}\right)$. Error bars represent the standard deviation of the measurements $(n=5)$.

while the green petals only generate moderate bending (Supplementary Movie 1). Another example is a wing flap of a photonic butterfly was demonstrated by patterning the photonic crystals to generate green butterfly wings with blue veins through selectively exposing part of the shaped bilayer to water vapor using stencils (Fig. 3d). When the structure is illuminated the wings close rapidly and subsequently open gradually after the light is switched off (Supplementary Movie 2).

The capacity to programmably pattern the photonic crystal layers by inducing molecular rearrangement in the protein matrix enables the regulation of local light propagation within the bilayer system. This approach allows for local optomechanical actuation to realize different motion modalities. To demonstrate this idea, the photonic lattices in the bilayers were patterned as shown in Fig. 3e-h. When SIO sides are illuminated by the laser beam, symmetric folding (Fig. 3e), outer bending (Fig. 3f), twisting (Fig. 3g), and asymmetric folding (Fig. $3 \mathrm{~h}$ ) are generated since only the patterned areas are significantly deformed because of the enhanced interaction between light and gold nanoparticles (see also Supplementary Movie 3). This is further verified by thermal imaging to observe temperature distribution during actuation. From the IR images, the patterned areas reach higher temperatures than the unpatterned areas. In addition to these macroscale patterning examples, it is possible to obtain finer patterning details and manipulate light on the microscale. As a demonstration, a series of microscale lines gradually changing from 50 to $200 \mu \mathrm{m}$ was designed on the photonic crystal, as shown in Fig. 3i, j. When this structure is illuminated, asymmetric bending is generated because of the heating gradient in response to the pattern (Fig. 3k). It is worth noting that water-vapor-based non-contact patterning method presented here allows for the creation of arbitrary, highresolution (few microns) patterns, of which the dimension should be determined according to the geometry of the photonic bilayer to achieve desired deformation.
Phototropic moving. While patterning photonic crystal layers effectively tailors the bandgap for controllable light manipulation, the angular dependence of the reflectivity (Supplementary Fig. 5a, b) allows for dynamic, reversible, and tunable optomechanical actuation. This is explored by evaluating the bilayer deformation at different illumination angles (Supplementary Fig. 2). Three actuation cases can be identified: (i) bilayer strip rotates perpendicularly to the short axis either away from (Fig. 4a) or (ii) towards (Fig. 4b) the light source, and (iii) the strip rotates perpendicularly to the long axis (Fig. 4c). The actuation of the photonic film with illumination angle is dramatically different from that of SF/PDMS film, especially for SIO side illumination whose displacement increases significantly at first and then decreases with the increase of illumination angle. Taking SIO side illumination of the photonic bilayer film in case (i) for instance, the displacement increases first until $70^{\circ}$ because of the gradually enhanced light absorption and photothermal conversion (Supplementary Fig. 5c) with the blueshift of the stop-band and then decreases quickly due to the largely reduced amounts of light on the sample.

This feature is reminiscent of the heliotropic bending and moving of sunflowers with sunlight ${ }^{44}$ and could provide inspiration for tether free, light responsive materials that adapt and reconfigure themselves in response to moving light. As a demonstration, a photonic sunflower with pedicel composed of a photonic bilayer film but petals and stamen composed of SIO was designed (Fig. 4d). The schematics and images shown in Fig. 4e, $\mathrm{f}$ show the artificial sunflower continuously tracks the movement of a light source while its petals and stamen keep facing the light because of the continuous bending of its pedicel (see also Supplementary Movie 4). When the irradiation ceases, the sunflower returns to its initial position. In addition to bending tracking, phototropic twisting motion can also be achieved by locally attaching PDMS on SIO (Supplementary Fig. 6a). The lollipop-like geometry tracks the light source continuously with a 


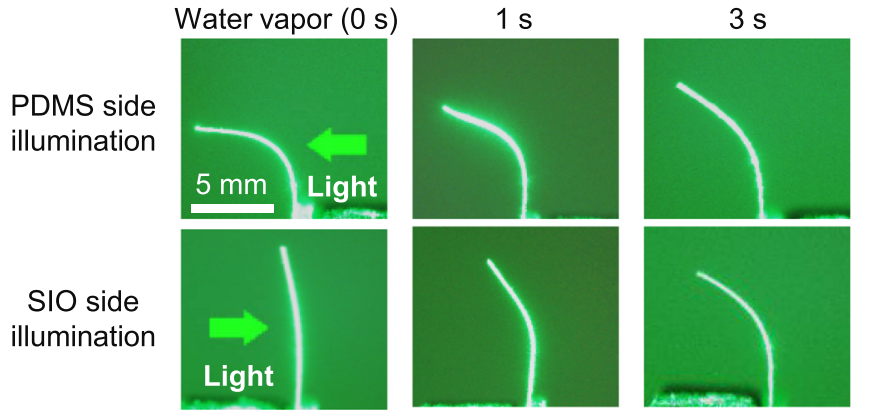

C
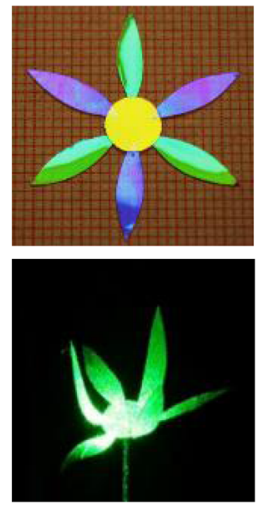

e
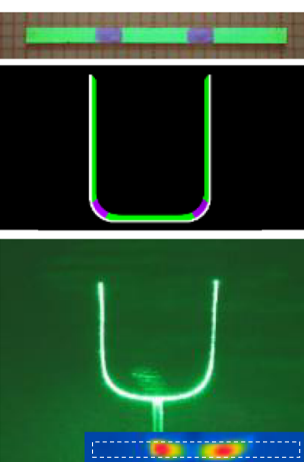

i

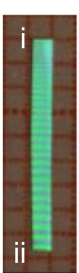

d
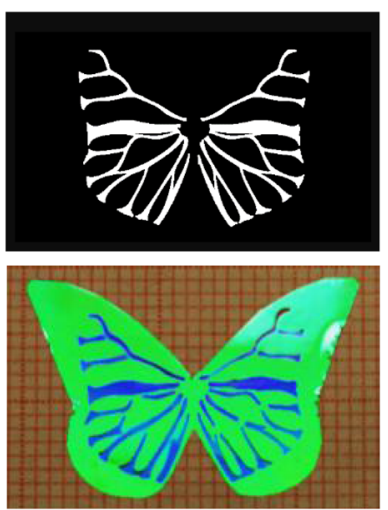

f
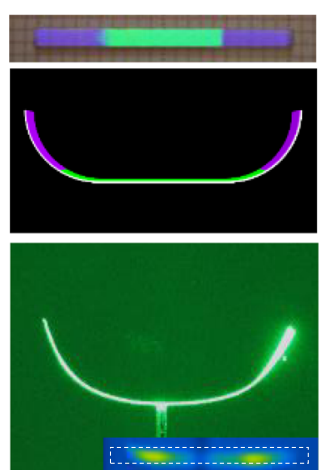

j

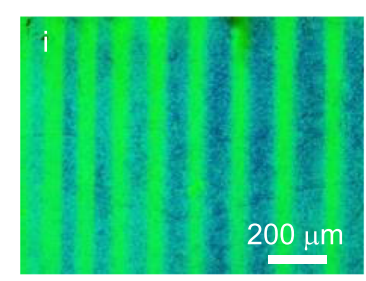

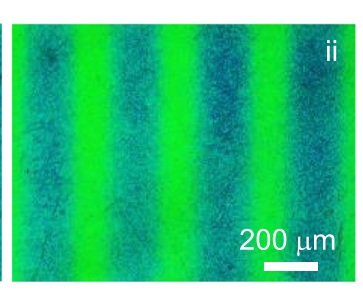

g
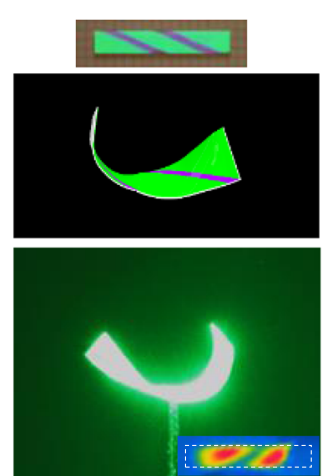

$\mathbf{k}$
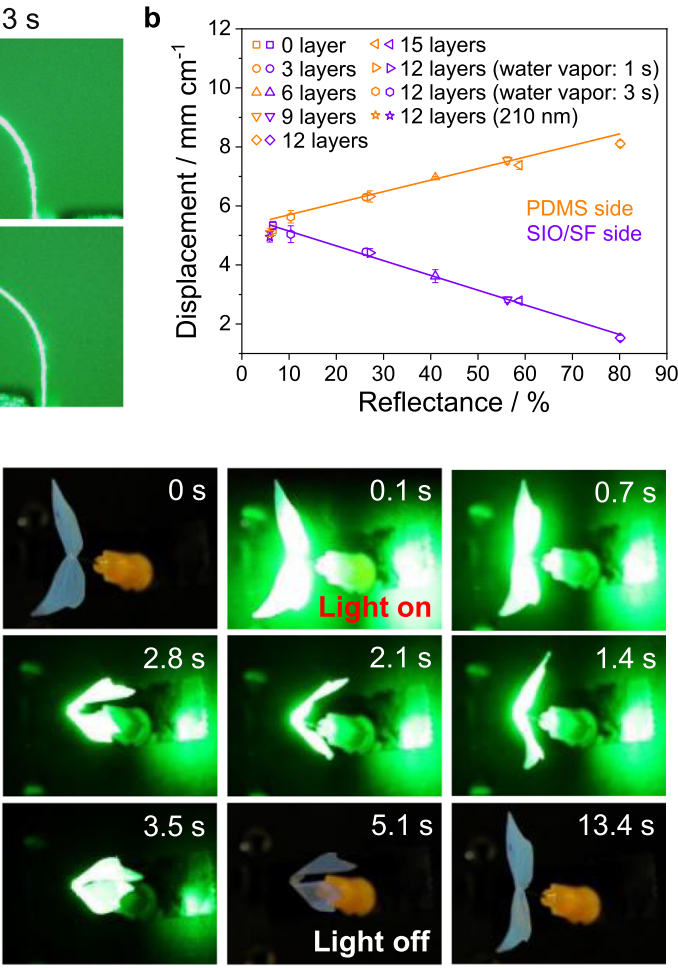

h
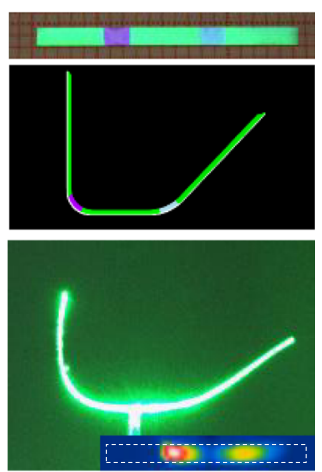

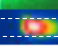
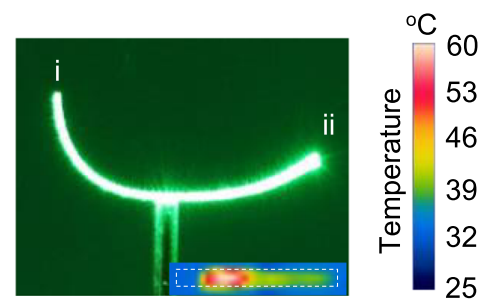

Fig. 3 Programmable motion via photonic nanostructure design. a Photographs showing the deformation of the same photonic actuator before and after water vapor treatment for different durations $\left(I=35 \mathrm{~m} \mathrm{~cm}^{-2}\right)$. b Dependence of displacement on the reflectance intensity of the $\mathrm{SIOs}$ at $532 \mathrm{~nm}(I=$ $35 \mathrm{~m} \mathrm{~cm}^{-2}$ ). c Photographs of a flower-shaped geometry (top) and relative selective bending of the photonic bilayer structure after light irradiation (bottom). d (Left) Stencil design used to produce a pattern on a butterfly-shaped photonic bilayer (top) and the corresponding photograph (bottom). The pattern was generated by exposing masked SIO to water vapor for $3 \mathrm{~s}$. (Right) The actuation process of the butterfly under laser illumination from PDMS side (Input power: $0.5 \mathrm{~W}$ ). e-h (Top) Photographs of patterned bilayer strips by water vapor exposure (e-g and $\mathbf{h}$-left: $3 \mathrm{~s}, \mathbf{h}$-right: $1 \mathrm{~s}$ ). The width of patterns is: $2 \mathrm{~mm}$ in $\mathbf{e}$ and $\mathbf{h}, 5 \mathrm{~mm}$ in $\mathbf{f}$, and $1 \mathrm{~mm}$ in $\mathbf{g}$. (Middle and bottom) Schematics and images of the corresponding motion modes under laser illumination from SIO side: symmetrical folding (e), outer bending (f), twisting ( $(\mathbf{g}$ ), and unsymmetrical folding (h). i Photograph of a bilayer strip with microscale lines. The line width changes gradually from one side to another. $\mathbf{j}$ Optical microscopy images showing the lines near the two margin areas of the strip. $\mathbf{k}$ Corresponding motion mode upon exposure to laser source. The insets in $\mathbf{e}-\mathbf{h}$ and $\mathbf{k}$ show the infrared images of the patterned strips under laser irradiation with dashed line outlining the edge of the sample before laser irradiation. Error bars denote the standard deviation of the measurements $(n=5)$.

twisting motion (Supplementary Fig. 6b, c, see also Supplementary Movie 5).

This wireless, light responsive, phototropic system has utility as a light tracking device and could be used to enhance light-to-energy conversion efficiency when interfaced to photosensitive systems. As a demonstration, an array of transfer-printed solar cells (weight: $\sim 20 \mu \mathrm{g}$ for each one) is integrated with the photonic bilayer film (Fig. 4g). The solar cell light conversion efficiency is 
a

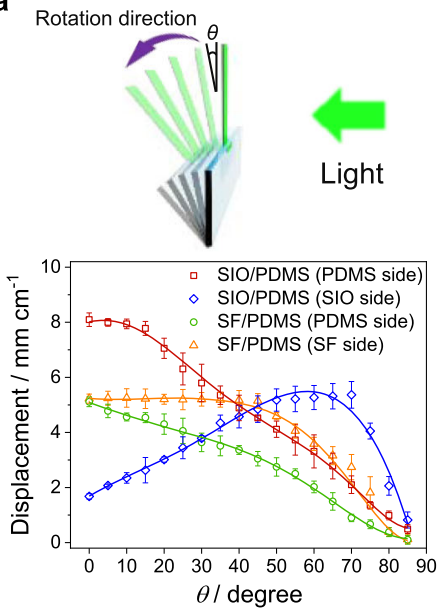

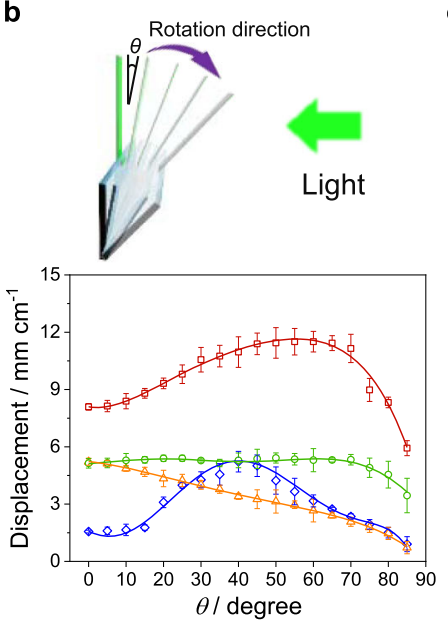

c

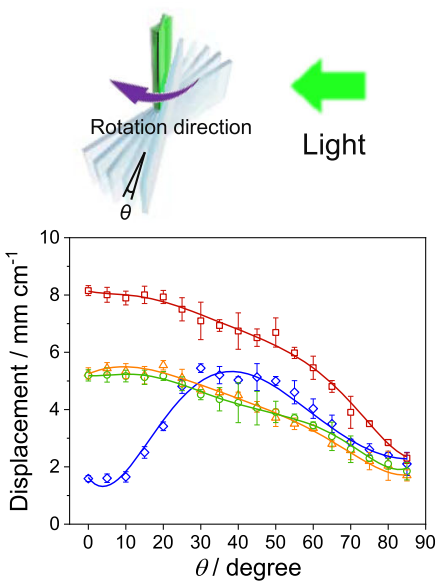

d
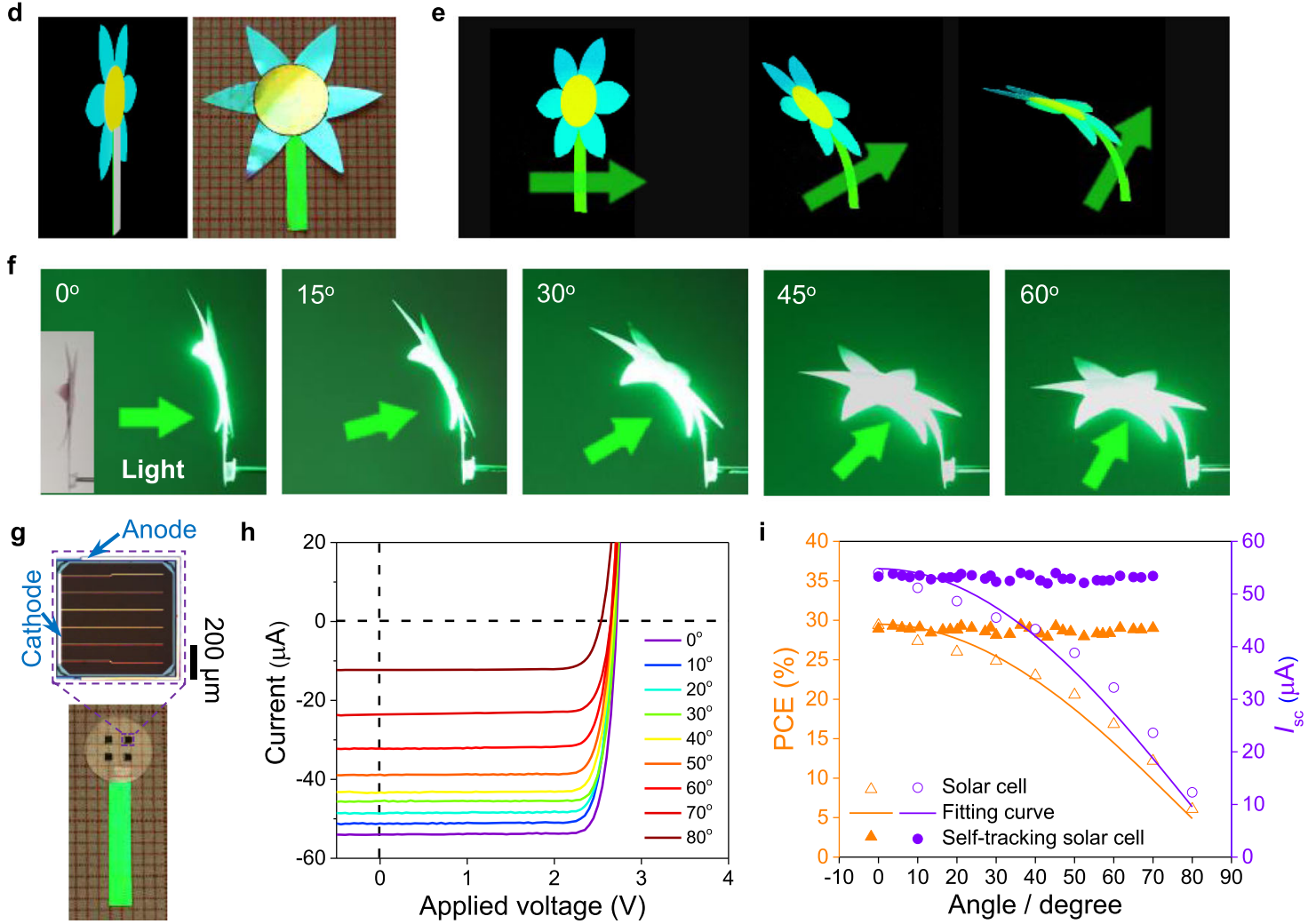

Fig. 4 Angle-dependent actuation and phototropic moving. a-c (Top) Schematics of the rotation direction of the sample with respect to the laser source. The sample was illuminated from either PDMS or SIO/SF side. (Bottom) Dependence of displacement of the bilayers on the rotation angle $(\theta)$ of the sample $\left(I=35 \mathrm{~m} \mathrm{~cm}^{-2}\right)$. Same legend for $\mathbf{a}, \mathbf{b}$, and $\mathbf{c}$. $\mathbf{d}$ Schematic (left) and photograph (right) of a photonic sunflower. e, $\mathbf{f}$ Schematics (e) and images (f) showing different deformation states of the artificial sunflower with different illumination angles $\left(I=35 \mathrm{~m} \mathrm{~cm}^{-2}\right)$. Insert in $\mathbf{f}$ indicates the initial state of the sunflower before illumination. $\mathbf{g}$ Photograph of the self-tracking photovoltaics system made of a photonic bilayer strip and an array of transfer-printed solar cells. Enlarged image shows the structure of one of the transfer-printed solar cells. $\mathbf{h}$ Current (I)-voltage (V) curves of the solar cell at different angles of incidence of light. i Measured power conversion efficiency (PCE) and short-circuit current $\left(I_{\mathrm{sc}}\right)$ as a function of incident angle. Error bars indicate the standard deviation $(n=5)$.

angle-dependent (Fig. $4 \mathrm{~h}$ and Supplementary Fig. 7), i.e., the power conversion efficiency (PCE) and short-circuit current $\left(I_{\mathrm{sc}}\right)$ decrease gradually as the illumination angle is increased with respect to the photoactive surface (Fig. 4i). After integration with the phototropic photonic film, the solar cells track the light source continuously (Supplementary Movie 6) keeping the angle between the solar cells' substrate and the laser beam nearly constant (Supplementary Fig. 8) and maximizing the efficiency of the solar cell as the light source moves. As shown in Fig. 4i, both PCE and $I_{\mathrm{sc}}$ of the self-tracking solar cells keep almost unchanged with varying the illumination angle. While this example is optimized for a specific wavelength, in a real-life scenario would have to be adapted to accommodate a flexible structural color filter ${ }^{45}$ or have a broad-band photonic structure $^{46}$ in order to achieve heliotropic movement under sunlight illumination. In addition, the weight and size of the device that can be mounted in such photonic bilayer are determined by its geometry, which should be further optimized to improve its loading capacity for devices. Phototropic structures such as this could be 
also integrated with other solar energy utilization systems/devices, such as solar thermal collectors or artificial photosynthesis devices, to adaptively optimize their energy output.

Reconfigurable actuation. Finally, given the confluence of reconfigurability of photonic structures and angle dependence of stop-band, the photonic actuators could provide interesting opportunities to alternate between different motion modes and realize switchable three-dimensional configurations by only tuning the illumination angle. The former is demonstrated by reexamining the motion modes of the patterned samples shown in Fig. 3e- $\mathrm{h}$ after being laterally exposed to laser beam (such as $45^{\circ}$ ). Interestingly, only overall bending is generated for all the samples because of the similar light capture within the patterned and unpatterned areas (Supplementary Fig. 9). The latter is demonstrated by reshaping the photonic bilayer into a cross-shaped geometry with two symmetric sides locally patterned using water vapor (Supplementary Fig. 10a). When the bilayer film is illuminated vertically from the SIO side $\left(0^{\circ}\right)$, only the patterned two sides fold (Supplementary Fig. 10b, Supplementary Movie 7), which is consistent with the anisotropic temperature distribution during actuation. However, if the sample is laterally exposed to laser beam $\left(45^{\circ}\right)$, the four sides fold simultaneously into a boxlike shape because of distributed heating of both patterned and unpatterned regions (Supplementary Fig. 10b, Supplementary Movie 7).

\section{Discussion}

The combination of a tunable biopolymer material (silk fibroin), an elastomer, and a reconfigurable photonic crystal structure provides complex modes of programmable optomechanical actuation by molding the flow of light into the composite structure. This strategy allows controlled spectral distribution at the microscale, offering the potential for photonic microactuators with complex deformation with potential utility for microrobotic technologies. The availability of a reconfigurable photonic crystal layer for effective, angle-dependent light harvesting opens up the possibility of generating optomechanical actuators that act both as back reflectors ${ }^{11,47}$ and solar trackers. The reconfigurability of the photonic crystal layer and the ease of functionalization of silk matrix can provide new avenues for optical devices with complex actuation by incorporating photothermal components with different absorption properties such as gold nanocrystals with different morphologies ${ }^{48}$ or carbon nanotube with selective chirality distributions ${ }^{32}$, and by selectively matching the wavelength of light source, photonic bandgap, and the absorption band. Considerable opportunities exist to expand this work to other optomechanical systems, like liquid-crystal elastomers, hydrogels, and shape memory polymers opening promising directions for future development of intelligent and multifunctional optomechanical devices.

\section{Methods}

Gold nanoparticles synthesis. Citrate stabilized gold nanoparticles were synthesized using the method described in literature ${ }^{49}$. Trisodium citrate dihydrate (S1804, Sigma-Aldrich, USA) and gold (III) chloride trihydrate (520918, SigmaAldrich, USA) were used without further modification. In a $50-\mathrm{mL}$ beaker, $20 \mathrm{~mL}$ of $1.0 \mathrm{mM} \mathrm{HAuCl}{ }_{4}$ aqueous solution was heated to boiling under stir. After the solution began to boil, $2 \mathrm{~mL}$ of $38.8 \mathrm{mM}$ trisodium citrate aqueous solution was quickly added to the beaker. In about 10 min with continued boiling and stirring, the solution became deep red color. During the boiling, the volume of the solution decreased due to evaporation, so that DI water was added to keep the total solution volume near $22 \mathrm{~mL}$. When the solution was a deep red color, the beaker was removed from the hotplate and allowed to cool to room temperature. The cooled solution was centrifuged at $17,500 \mathrm{rpm}$ for $30 \mathrm{~min}$ or until supernatant is clear. The supernatant was discarded, and $10 \mathrm{~mL}$ DI water was added to the tube to make AuNPs stock solution. The prepared solution was sonicated for $20 \mathrm{~min}$ before use each time to suspend the nanoparticles fully.
}

Gold-nanoparticles-doped silk solution preparation. The regenerated aqueous silk fibroin solution was prepared using the established protocols ${ }^{50}$. Bombyx mori silk cocoons were boiled in a $0.02 \mathrm{M} \mathrm{Na}_{2} \mathrm{CO}_{3}$ water solution for 30 min to remove the sericin. The remaining fibroin was then rinsed in deionized water and allowed to dry for 2 days. The dried fibroin was dissolved in $9.3 \mathrm{M} \mathrm{LiBr}$ solution at $60^{\circ} \mathrm{C}$ for $4 \mathrm{~h}$, followed by a dialysis process against deionized water for 3 days to obtain a 7-8 $\mathrm{wt} \%$ silk fibroin solution in water. Gold-nanoparticles-doped silk solutions were prepared by mixing as-prepared silk solution with $150 \mu \mathrm{L}$ gold nanoparticles solution. Gentle agitation was applied to obtain a uniform dispersion.

Gold-nanoparticles-doped silk inverse opal (SIO) films preparation. The goldnanoparticles-doped SIO films were prepared by using polystyrene colloidal crystal multilayers as templates. The detailed fabrication method was described in refs. ${ }^{38,39,46}$ and reproduced here in summary. Briefly, $30 \mu \mathrm{L}$ suspension (4\%) of monodisperse polystyrene spheres (with diameter of $210 \mathrm{~nm}$ or $300 \mathrm{~nm}$ ) was introduced to the water surface to form a floating monolayer. After removing the spheres immersed into the subphase of water, large-scale hexagonally close-packed polystyrene monolayer array was formed at the water/air interface with the aid of sodium dodecyl sulfate. This monolayer was then transferred from the water surface to the surface of a polymethyl methacrylate-precoated $\mathrm{Si}$ wafer. By repeating the transferring procedure, colloidal crystal multilayers with desired layer numbers were obtained. The silk solution doped with gold nanoparticles was carefully cast onto the colloidal crystal multilayers to fill all the air voids. The sample was left to dry for $12 \mathrm{~h}$ (at $25^{\circ} \mathrm{C}, 30-40 \%$ relative humidity) to form a composite film. The goldnanoparticles-doped SIO films with thickness of $\sim 15 \mu \mathrm{m}$ were finally obtained after removing the polystyrene templates in toluene. Gold-nanoparticles-doped silk flat films with thickness of $\sim 15 \mu \mathrm{m}$ were also prepared as control samples.

Fabrication of bilayer films. The bilayer films were prepared by casting PDMS (base agent and cure agent are of 10:1 weight ratio) onto the SIO (non-nanostructured surface) or SF film via spin coating $(1300 \mathrm{rpm} \times 30 \mathrm{~min})$ and then allowed to cure at $60^{\circ} \mathrm{C}$ for $12 \mathrm{~h}$. The thickness of the PDMS is $\sim 60 \mu \mathrm{m}$.

Fabrication of patterned SIO/PDMS bilayer films. Water vapor treatment was used to design various patterns. Stencils with various designs and sizes were first positioned on the surface of the SIO/PDMS films from the SIO side. Then, the masked bilayer films were placed above a heated water surface (about $40^{\circ} \mathrm{C}$ ) to directly expose the SIO surface to water vapor for a set of different durations. The distance between the films and water surface was fixed at $5 \mathrm{~mm}$.

Fabrication of solar cells. With a sacrificial layer of AlInP to aid the epitaxial liftoff (ELO) processes, a lattice-matched tandem structure of InGaP/GaAs/ InGaAsNSb was epitaxially grown on a GaAs substrate to build printable threejunction ( $3 \mathrm{~J}$ ) solar $\mu$-cells ${ }^{51}$. Photolithography and wet etching processes define an active area of $650 \times 650 \mu \mathrm{m}^{2}$ for the $3 \mathrm{~J}$ cells and create trenches among cells for ELO process. Then, metal deposition of $\mathrm{Ge} / \mathrm{Ni} / \mathrm{Au}$ layers formed metal grid/fingers for cathode and anode contacts in recessed geometries. A patterned photoresist (8 $\mu \mathrm{m})$ across the trenches served as anchors and etching barrier during the ELO process, where the AlInP sacrificial layer was removed by hydrochloric acid to release the $3 \mathrm{~J}$ cells from the substrate. Microscale transfer printing utilizing a patterned elastomeric stamp enables the integration of $3 \mathrm{~J}$ cells onto PDMS substrate $^{52}$. Acetone rinsing and plasma aching was finally utilized to remove the photoresist anchors/residues.

Structural and properties characterization. FESEM (Zeiss Supra55VP) was used to observe the surface and cross-sectional morphology of the bilayer film. TEM (JEOL, 2100) was used to observe the morphology of gold nanoparticles. The reflectance spectra were recorded using a fiber-optic spectrometer (USB-2000, Ocean Optics). The absorption spectrum of gold nanoparticles was measured with a spectrophotometer (V-570, Jasco). Photographs and movies were taken using a DSLR camera (Canon EOS rebel T1i). Temperatures and IR thermal images were recorded using an infrared camera (SC645, FLIR Systems, Inc., Sweden). The spatial maximum temperature across the whole sample was recorded and averaged over a one minute period after the temperature reading was stabilized. For each laser intensity, three samples were measured and the mean value of their temporally averaged temperatures was calculated. The thermal degradation experiment was performed with a thermogravimetric analyzer (Q500, TA Instruments, USA) at a heating rate of $20 \mathrm{~K} / \mathrm{min}$. Specific heat capacity was measured with a differential scanning calorimeter (Q100, TA Instruments, USA) at a heating rate of $20 \mathrm{~K} / \mathrm{min}$ For the performance characterization of solar cells, thin film metallization of $\mathrm{Cu}$ followed by photolithographic patterning was performed to establish the terminal interconnects and pads for probing. The printed $3 \mathrm{~J}$ cells were characterized by a Keithley 2400 source meter under AM1.5 G illumination with an irradiation intensity of $100 \mathrm{~mW} \mathrm{~cm}^{-2}$. A manual tilt stage facilitates the angular dependent analysis of cell performance, where the angle of incidence is determined by the calibrated tilt with respect to the solar simulator (Newport). The photovoltaic properties of self-tracking solar cell at different illumination angles were measured by leveraging the angle value between the solar cells' substrate and the laser beam at corresponding illumination angle. 
Displacement measurement and data analysis. The tested samples had their edges taped onto a glass slide which was fixed to an optical mount. The illumination area of a collimated green laser with wavelength of $532 \mathrm{~nm}$ (Millennia Pro ${ }^{\mathrm{nw}}$, Spectra-Physics, USA) was expanded by a lens to cover the entire sample. The beam waist was measured by the knife-edge method. For actuator displacement analysis the beam waist radius was $4.32 \mathrm{~mm}$. The total output power $P_{\text {total }}$ of the laser was measured by an optical power meter (PM100A, Thorlabs GmbH, Germany). The received power (or input power, $P_{\text {input }}$ ) on the sample surface from a Gaussian laser beam was calculated by:

$$
P_{\text {input }}=(1-R) \int_{-w / 2}^{w / 2} \int_{-l / 2}^{l / 2} I_{0} \exp \left(-\frac{2\left(x^{2}+y^{2}\right)}{r^{2}}\right) d x d y
$$

where $R$ is the reflectance of the sample surface, $r$ is the beam radius, $I_{0}$ is the intensity amplitude of the laser calculated by $I_{0}=2 P_{\text {total }} / \pi r^{2}$ for Gaussian beam profile, $w$ and $l$ are the sample width and length, respectively.

The illumination intensity $(I)$ was calculated by:

$$
I=P_{\text {input }} / A
$$

where $A$ is the area of the sample.

The deformation of the bilayer during actuation was recorded by a DSLR camera (Canon EOS rebel T1i) at a frame rate of $30 \mathrm{fps}$ and the tip movement was analyzed using Matlab ${ }^{\circ}$. The length of the samples $(1 \mathrm{~cm})$ was used to calibrate the pixel length in order to calculate the displacement. All the displacement measurements were performed at $20-25^{\circ} \mathrm{C}$ and $20-30 \% \mathrm{RH}$.

Optical simulation. The numerical simulation of the SIOs was carried out using rigorous coupled-wave analysis (RCWA ${ }^{38}$. We considered a 12-layer SIO consisting of a silk matrix with spherical air holes lying on a substrate made of silk and gold nanoparticles. The presence of gold nanoparticles was taken into account by means of an effective dielectric function calculated using the Maxwell-Garnett model, in which the porosity corresponds to the concentration of the gold nanoparticles dispersed inside the silk matrix. The theoretical reflectance spectrum was then fitted to the experimental data by taking the lattice constant $\Lambda$ as the only free parameter, leading to an estimated value $\Lambda=282 \mathrm{~nm}$. Since in the experiment we used non-polarized light, the theoretical spectra are obtained by averaging TE and TM reflectance spectra, which have been calculated for angles of incidence in the range from $0^{\circ}$ to $45^{\circ}$ with an interval step of $5^{\circ}$.

Calculation of photothermal conversion efficiency. The method used for calculating photothermal conversion efficiency is elaborated in refs. $28,53,54$ which is briefly introduced here. The photothermal conversion efficiency is defined as the ratio of thermal energy $Q$ to input light energy $q$ :

$$
\eta=\frac{Q}{q}
$$

The generated heat $Q$ raises the sample temperature to $T_{\max }$ from environmental temperature $T_{\text {room }}^{\max }$ and can be expressed by:

$$
Q=U A\left(T_{\max }-T_{\text {room }}^{\max }\right)
$$

where $U$ is heat transfer coefficient, $A$ is the sample surface area, and $T_{\text {room }}^{\max }$ refers to the maximum air temperature surrounding the sample. Rate constant of the energy loss is defined as:

$$
w=\frac{U A}{C m}
$$

where $C$ is mass-specific heat capacity and $m$ is the mass of the sample.

By fitting the cooling curve of the experimental results with:

$$
T(t)=\left(T_{\max }-T_{\text {room }}-a\right) \exp (-w t)+a \cdot \exp \left(-w_{\text {surr }} t\right)+T_{\text {room }}
$$

with coefficient $a=\frac{w}{w-w_{\text {surr }}}\left(T_{\text {room }}^{\max }-T_{\text {room }}\right)$

where $w_{\text {surr }}$ is the rate constant of the air surrounding the sample, and $T_{\text {room }}$ is the air temperature surrounding the sample at $t=0$ (i.e., room temperature), one can obtain the value for $w, w_{\text {surr }}$, and $T_{\text {room }}^{\max }$. With known values for $C$ and $m$, one can calculate the values for $U$ and $Q$, and further acquire the photothermal conversion efficiency $\eta$.

Calculation of angle-dependent photovoltaic properties. The open voltage is dependent on the incident angle with the following relation ${ }^{55}$

$$
\begin{aligned}
V_{\mathrm{oc}(\theta)} & =V_{0}+C \ln (\cos \theta) \\
C & =\frac{m k T}{e}
\end{aligned}
$$

where $V_{0}$ is the open circuit voltage at normal incidence, $m$ is the non-ideal diode factor, $k$ is the Boltzman's constant, $T$ is the temperature of the solar cell, and $e$ is the electron charge. By fitting the $V_{o c}$ data with different incident angles, we obtained the $V_{0}$ value to be $(2.698 \pm 0.023) \mathrm{V}$ and $C$ is fitted to be $(0.080 \pm 0.03) \mathrm{V}$. The error bar is $95 \%$ confidence interval for all the fitting processes. The solar cell efficiency $(\eta)$ also known as the power conversion efficiency (PCE) is defined as the ratio of power generated by the cell and the input light power. The generated power can be calculated with open circuit voltage $V_{\mathrm{oc}}$, short circuit current $I_{\mathrm{sc}}$, and fill factor $F F$.

$$
\eta=\frac{V_{\mathrm{oc}} I_{\mathrm{sc}} F F}{P_{\mathrm{in}}}
$$

$F F$ is independent of incident angle, while $I_{\mathrm{sc}}$ obeys Lambert's cosine law ${ }^{56}$, thus

$$
I_{\mathrm{sc}}(\theta)=I_{0} \cos \theta
$$

where $I_{0}$ is the short circuit current with normal light incidence. With both $V_{\mathrm{oc}}$ and $I_{\mathrm{sc}}$ being angle dependent, the efficiency can be written as

$$
\eta(\theta)=\frac{V_{\mathrm{oc}}(\theta) I_{\mathrm{sc}}(\theta) F F}{P_{\mathrm{in}}}=\eta_{0} \cos \theta+\eta_{0} \frac{1}{V_{0}} C \ln (\cos \theta) \cos \theta
$$

By fitting the $\boldsymbol{\eta}(\boldsymbol{\theta})$ data, one obtains $\boldsymbol{\eta}_{0}=(29.5 \pm 1.74)$.

\section{Data availability}

The data that support the findings of this study are available within the main text and the Supplementary Information. All data are also available from the corresponding authors upon reasonable request.

Received: 14 November 2020; Accepted: 28 January 2021; Published online: 12 March 2021

\section{References}

1. Vukusic, P. \& Sambles, J. R. Photonic structures in biology. Nature $\mathbf{4 2 4}$ 852-855 (2003).

2. Tadepalli, S., Slocik, J. M., Gupta, M. K., Naik, R. R. \& Singamaneni, S. Biooptics and bio-inspired optical materials. Chem. Rev. 117, 12705-12763 (2017).

3. Yu, K. L., Fan, T. X., Lou, S. \& Zhang, D. Biomimetic optical materials: integration of nature's design for manipulation of light. Prog. Mater. Sci. 58, 825-873 (2013)

4. Gur, D., Palmer, B. A., Weiner, S. \& Addadi, L. Light manipulation by guanine crystals in organisms: biogenic scatterers, mirrors, multilayer reflectors and photonic crystals. Adv. Funct. Mater. 27, 1603514 (2017).

5. Schroeder, T. B. H., Houghtaling, J., Wilts, B. D. \& Mayer, M. It's not a bug, it's a feature: functional materials in insects. Adv. Mater. 30, 1705322 (2018)

6. Zhou, H. et al. Bio-inspired photonic materials: prototypes and structural effect designs for applications in solar energy manipulation. Adv. Funct. Mater. 28, 1705309 (2018).

7. Yablonovitch, E. Inhibited spontaneous emission in solid-state physics and electronics. Phys. Rev. Lett. 58, 2059-2062 (1987).

8. John, S. Strong localization of photons in certain disordered dielectric superlattices. Phys. Rev. Lett. 58, 2486-2489 (1987).

9. Liu, J. et al. Slow photons for photocatalysis and photovoltaics. Adv. Mater. 29, 1605349 (2017)

10. O’Brien, P. G. et al. Enhanced photothermal reduction of gaseous $\mathrm{CO} 2$ over silicon photonic crystal supported ruthenium at ambient temperature. Energy Environ. Sci. 11, 3443-3451 (2018).

11. O'Brien, P. G. et al. Silicon photovoltaics using conducting photonic crystal back-reflectors. Adv. Mater. 20, 1577-1582 (2008).

12. Lodahl, P. et al. Controlling the dynamics of spontaneous emission from quantum dots by photonic crystals. Nature 430, 654-657 (2004).

13. Lee, J., Min, K., Park, Y., Cho, K. S. \& Jeon, H. Photonic crystal phosphors integrated on a blue LED chip for efficient white light generation. Adv. Mater 30, 1703506 (2018)

14. Sanchez-Sobrado, O. et al. Interplay of resonant cavity modes with localized surface plasmons: optical absorption properties of Bragg stacks integrating gold nanoparticles. Adv. Mater. 23, 2108-2112 (2011).

15. Kim, S. et al. Silk inverse opals. Nat. Photon 6, 817-822 (2012).

16. Rich, S. I., Wood, R. J. \& Majidi, C. Untethered soft robotics. Nat. Electron. 1, 102-112 (2018)

17. Palagi, S. \& Fischer, P. Bioinspired microrobots. Nat. Rev. Mater. 3, 113-124 (2018).

18. Wu, Z. G. et al. Near-infrared light-triggered "On/Off" motion of polymer multi layer rockets. ACS Nano 8, 6097-6105 (2014). 
19. Xie, H. H. et al. Biodegradable near-infrared-photoresponsive shape memory implants based on black phosphorus nanofillers. Biomaterials 164, 11-21 (2018).

20. Zhang, L. D., Liang, H. R., Jacob, J. \& Naumov, P. Photogated humidity-driven motility. Nat. Commun. 6, 7429 (2015).

21. Sitti, M. \& Wiersma, D. S. Pros and cons: magnetic versus optical microrobots. Adv. Mater. 32, 1906766 (2020).

22. Gelebart, A. H. et al. Making waves in a photoactive polymer film. Nature 546, 632-636 (2017).

23. Gelebart, A. H., Mulder, D. J., Vantomme, G., Schenning, A. P. H. J. \& Broer, D. J. A rewritable, reprogrammable, dual light-responsive polymer actuator. Angew. Chem. Int. Ed. 56, 13436-13439 (2017).

24. Zeng, H. et al. Light-fuelled freestyle self-oscillators. Nat. Commun. 10, 5057 (2019).

25. Zhao, Y. S. et al. Soft phototactic swimmer based on self-sustained hydrogel oscillator. Sci. Robot. 4, eaax7112 (2019).

26. Qian, X. et al. Artificial phototropism for omnidirectional tracking and harvesting of light. Nat. Nanotechnol. 14, 1048-1055 (2019).

27. Jin, B. J. et al. Programming a crystalline shape memory polymer network with thermo- and photo-reversible bonds toward a single-component soft robot. Sci. Adv. 4, eaao3865 (2018).

28. Yang, H. et al. $3 \mathrm{D}$ printed photoresponsive devices based on shape memory composites. Adv. Mater. 29, 1701627 (2017).

29. Tai, Y. L., Lubineau, G. \& Yang, Z. G. Light-activated rapid-response polyvinylidene-fluoride-based flexible films. Adv. Mater. 28, 4665-4670 (2016).

30. Liu, Y., Shaw, B., Dickey, M. D. \& Genzer, J. Sequential self-folding of polymer sheets. Sci. Adv. 3, e1602417 (2017).

31. $\mathrm{Mu}, \mathrm{J}$. K. et al. Origami-inspired active graphene-based paper for programmable instant self-folding walking devices. Sci. Adv. 1, e1500533 (2015).

32. Zhang, X. et al. Photoactuators and motors based on carbon nanotubes with selective chirality distributions. Nat. Commun. 5, 2983 (2014).

33. Li, C. et al. Supramolecular-covalent hybrid polymers for light-activated mechanical actuation. Nat. Mater. 19, 900-909 (2020).

34. Palagi, S. et al. Structured light enables biomimetic swimming and versatile locomotion of photoresponsive soft microrobots. Nat. Mater. 15, 647-653 (2016).

35. Shahsavan, H. et al. Bioinspired underwater locomotion of light-driven liquid crystal gels. Proc. Natl Acad. Sci. USA 117, 5125-5133 (2020).

36. Li, C. S., Liu, Y., Huang, X. Z. \& Jiang, H. R. Direct sun-driven artificial heliotropism for solar energy harvesting based on a photo-thermomechanical liquid-crystal elastomer nanocomposite. Adv. Funct. Mater. 22, 5166-5174 (2012).

37. Wang, T., Torres, D., Fernandez, F. E., Wang, C. \& Sepulveda, N. Maximizing the performance of photothermal actuators by combining smart materials with supplementary advantages. Sci. Adv. 3, e1602697 (2017).

38. Wang, Y. et al. Modulation of multiscale 3D lattices through conformational control: painting silk inverse opals with water and light. Adv. Mater. 29, 1702769 (2017).

39. Wang, Y. et al. Biomaterial-based "Structured Opals" with programmable combination of diffractive optical elements and photonic bandgap effects. Adv. Mater. 31, 1805312 (2019).

40. Saravanan, D. Spider silk - structure, properties and spinning. J. Text. Appar., Technol. Manag. 5, 1-20 (2006).

41. Hu, Y. et al. A graphene-based bimorph structure for design of high performance photoactuators. Adv. Mater. 27, 7867-7873 (2015).

42. Vogel, N., Goerres, S., Landfester, K. \& Weiss, C. K. A convenient method to produce close- and non-close-packed monolayers using direct assembly at the air-water interface and subsequent plasma-induced size reduction. Macromol. Chem. Phys. 212, 1719-1734 (2011).

43. Oh, J. R., Moon, J. H., Yoon, S., Park, C. R. \& Do, Y. R. Fabrication of waferscale polystyrene photonic crystal multilayers via the layer-by-layer scooping transfer technique. J. Mater. Chem. 21, 14167-14172 (2011).

44. Atamian, H. S. et al. Circadian regulation of sunflower heliotropism, floral orientation, and pollinator visits. Science 353, 587-590 (2016).

45. Kwon, H. \& Kim, S. Chemically tunable, biocompatible, and cost-effective metal-insulator-metal resonators using silk protein and ultrathin silver films. ACS Photonics 2, 1675-1680 (2015).

46. Wang, Y., Li, M., Colusso, E., Li, W. Y. \& Omenetto, F. G. Designing the Iridescences of biopolymers by assembly of photonic crystal superlattices. $A d v$. Opt. Mater. 6, 1800066 (2018).
47. Liu, Y. M. et al. Self-organized cholesteric liquid crystal polymer films with tunable photonic band gap as transparent and flexible back-reflectors for dyesensitized solar cells. Nano Energy 26, 648-656 (2016).

48. Lan, T., Hu, Y., Wu, G., Tao, X. \& Chen, W. Wavelength-selective and rebound-able bimorph photoactuator driven by a dynamic mass transport process. J. Mater. Chem. C. 3, 1888-1892 (2015).

49. McFarland, A. D., Haynes, C. L., Mirkin, C. A., Van Duyne, R. P. \& Godwin, H. A. Color my nanoworld. J. Chem. Educ. 81, 544A (2004).

50. Rockwood, D. N. et al. Materials fabrication from Bombyx mori silk fibroin. Nat. Protoc. 6, 1612-1631 (2011).

51. Yoon, J. et al. GaAs photovoltaics and optoelectronics using releasable multilayer epitaxial assemblies. Nature 465, 329-333 (2010).

52. Chang, J. K. et al. Biodegradable electronic systems in 3D, heterogeneously integrated formats. Adv. Mater. 30, 1704955 (2018).

53. Wang, J. et al. High-performance photothermal conversion of narrowbandgap $\mathrm{Ti}_{2} \mathrm{O}_{3}$ nanoparticles. Adv. Mater. 29, 1603730 (2017).

54. Li, M. et al. Flexible magnetic composites for light-controlled actuation and interfaces. Proc. Natl Acad. Sci. USA 115, 8119-8124 (2018).

55. Balenzategui, J. L. \& Chenlo, F. Measurement and analysis of angular response of bare and encapsulated silicon solar cells. Sol. Energy Mater. Sol. Cells 86, 53-83 (2005).

56. McCluney, W. R. Introduction to Radiometry and Photometry (Artech House, 1994).

\section{Acknowledgements}

The authors acknowledge support from the Office of Naval Research Grant (N00014-19 1-2399) for this work. The SEM measurements were performed in Harvard University Center for Nanoscale Systems (CNS), which is a member of the National Nanotechnology Coordinated Infrastructure Network (NNCI), which is supported by the National Science Foundation under NSF award No. 1541959.

\section{Author contributions}

Y.W. and F.G.O. conceived the concept and designed the research. Y.W., M.L., W.L., and B.J.K. performed the photonic actuator studies. J.-K.C., J.H.K., and J.A.R. performed the photovoltaic device studies. D.A. and M.a.L. executed the optical simulations. All authors contributed to data analysis. Y.W. and F.G.O. wrote the manuscript.

\section{Competing interests}

The authors declare no competing interests.

\section{Additional information}

Supplementary information The online version contains supplementary material available at https://doi.org/10.1038/s41467-021-21764-6.

Correspondence and requests for materials should be addressed to F.G.O.

Peer review information Nature Communications thanks the anonymous reviewers for their contribution to the peer review of this work.

Reprints and permission information is available at http://www.nature.com/reprints

Publisher's note Springer Nature remains neutral with regard to jurisdictional claims in published maps and institutional affiliations.

Open Access This article is licensed under a Creative Commons Attribution 4.0 International License, which permits use, sharing, adaptation, distribution and reproduction in any medium or format, as long as you give appropriate credit to the original author(s) and the source, provide a link to the Creative Commons license, and indicate if changes were made. The images or other third party material in this article are included in the article's Creative Commons license, unless indicated otherwise in a credit line to the material. If material is not included in the article's Creative Commons license and your intended use is not permitted by statutory regulation or exceeds the permitted use, you will need to obtain permission directly from the copyright holder. To view a copy of this license, visit http://creativecommons.org/ licenses/by/4.0/.

(C) The Author(s) 2021 